\title{
RESEARCH PAPER \\ THE IMPORTANCE OF TRADITIONAL HEALERS IN THE PLANNING OF RURAL HEALTHCARE DELIVERY IN GHANA: THE CASE OF BONE-SETTING SERVICES IN LOAGRI AND WUNGU
}

\author{
R. Adamtey, C. Y. Oduro and K. A. Ocloo \\ Department of Planning, KNUST, Kumasi \\ radamtey@yahoo.com/radamteysekade@gmail.com \\ Yawoduro68@gmail.com \\ afiocloo@yahoo.com
}

\begin{abstract}
There has been increasing debate on whether traditional healers actually matter in planning for healthcare delivery and how their services can be effectively integrated into planning for formal health delivery systems. Whilst many have raised issues of standardization and efficacy against traditional healing, others have acclaimed its efficacy and many Ghanaians are claimed to have confidence in it and use traditional healing simultaneously with orthodox treatment. Detailed qualitative interviews were held with two bone-setters, one in Loagri and one in Wungu, 150 bone patients, 100 community members and 20 health staff. It was revealed that rural healthcare planning must incorporate traditional bone-setters and therefore traditional healers as they offer useful services and are popular because of the proximity one has to such services in relation to clinics and hospitals, the cost of healthcare, socio-cultural beliefs and norms and the confidence the people with bone injuries have in traditional bone-setters. In spite of the importance of traditional bone-setters, the weak collaboration with district hospitals, inadequate accommodation, limited use of modern equipment, and inadequate support and recognition from the District Assembly and the Ministry of Health undermine their role. Effective collaboration between traditional bone-setters and the West Mamprusi District Hospital and the integration of traditional bone-setting into the national public health framework need urgent policy attention.
\end{abstract}

Keywords: Bone-setting, Ghana, planning, healthcare, traditional healers

\section{INTRODUCTION}

Access to quality healthcare is a major determinant of the productivity of nations, and also a fundamental human right of every individual irrespective of their socio-economic background and geographic location (UNDP, 2005).
Ghana's health policy upholds the principle that "health is not only a human right issue but also a key driver of development; and ultimately wealth creation" (Republic of Ghana, 1997; 2005a; 2007). It is therefore mandatory for governments to ensure that all citizens have access 


\section{Adamtey et al.}

to acceptable quality of basic healthcare services. There are, however, numerous challenges facing the healthcare system in Ghana especially in the rural areas. Insufficient and poor spatial distribution of health facilities, inadequate number of the already ill-equipped health facilities, limited access to public health services, high cost of healthcare due to weaknesses in health financing through poor insurance arrangements, and inadequate trained personnel at the facilities altogether make the modern healthcare system not capable of meeting the healthcare needs of Ghanaians especially those living in rural areas (Marcel et. al, 2007; Darimani, 2007; Republic of Ghana, 1997).

Because of the numerous problems with access to healthcare, a number of people resort to alternative means of treatment particularly traditional or indigenous healing which is used in this article to mean bone-setting. According to the WHO (2002: 1), about $80 \%$ of Africa's population uses traditional healing to help meet their healthcare needs. Traditional healers are therefore important stakeholders of the healthcare system in many parts of the developing world (WHO, 2002; Olayiwola, 1987). As noted by Olayiwola (1987: 177) "if there is to be any real improvement in the health of the underserved population of the world, there will have to be full utilisation of all available resources." This is fundamental to the primary healthcare approach. Traditional practitioners constitute the most abundant and, in many cases, valuable resources present in the community. In fact evidence suggests that many Ghanaians patronise both indigenous and orthodox health facilities simultaneously (see Republic of Ghana, 2005b; Kavi et. al., 2000).

Traditional or indigenous treatment is defined by Osemwenkha (2000:586) as "the sum total of all knowledge and practices whether explicable or not, used in the diagnosis, prevention and elimination of physical, mental and social imbalance and relying exclusively on practical experience and observations handed down from generation to generation, whether verbally or in writing." According to the WHO (2002: 7) "it encompasses ancient and culture-bound healthcare practices which existed before the application of science to health matters." This type of health service delivery relies exclusively on practical experience through apprenticeship and observations handed down from generation to generation whether verbally or in writing. As noted by Agarwal and Agarwal (2010), traditional bone-setters' proficiency in bone-setting is passed from generation to generation without any formal documentation whatsoever.

A very important component of traditional healing is bone-setting ( $\mathrm{MoH}, 1995)$. Bonesetters play a substantial role in the Ghanaian healthcare system. A traditional bone-setter takes care of sprains, dislocations, simple to complex fractures which mostly are emergency cases when they occur and require urgent attention and treatment. How swiftly patients with bone injuries are attended to might depend on a number of factors such as how close one is to a health facility, the ability to pay for the cost of treatment, means of transport to a health facility and the waiting time before one is attended to (Bukar et. al., 2009).

Although bone-setting has been in existence for more than 3000 years (Agarwal and Agarwal, 2010) and seems to be very important in healthcare delivery system in many countries, it appears that it has not received adequate scholarly attention in healthcare research, health policy, and overall planning for healthcare delivery. This is evident from the annual reports of both Ghana Health Service (GHS) and Ministry of Health $(\mathrm{MoH})$ for the years 2010 to 2012. None of these key health delivery documents contain anything on bone-setting. In addition, the reports on programme of Work of Ghana Health Service for 2010 and 2012 appear to overlook the role of bone-setting. The possible reasons for their neglect include the unorganised nature of bone-setters, lack of confidence of orthodox medical practitioners in the bone-setters and inadequate information that healthcare planners 
have about the work and capabilities of bonesetters in delivering healthcare services to the populace (Darimani, 2007).

Questions around the popularity and reliability of bone-setters are rooted in overall concerns raised about the efficacy of traditional healing in general. For example, the WHO (2002: 31) has noted that many allopathic medicine professionals, even those in countries with strong history of traditional medicine, express strong reservations and often frank disbelief about the purported benefits of traditional healing. This concern is informed by traditional healers' inability to answer questions around safety and efficacy. The fact that the practice is shrouded in secrecy and superstition makes it fail the test of scientific inquiry. In spite of these concerns, traditional healing has been accepted as a reliable complement to modern healthcare.
So how do bone-setters complement the services provided by the allopathic practitioners in hospitals particularly in highly deprived rural regions in the country which are characterised by difficult accessibility? Do these services help us understand alternative means to approach rural healthcare planning in Ghana?

\section{STUDY METHODOLOGY}

The primary data collection was carried out from February to April of 2013 in Loagri and Wungu in the West Mamprusi District of the Northern Region of Ghana (Fig. 1).

Both communities were chosen because they are rural. These communities are noted for having renowned traditional bone-setters. The bone -setters in these two communities focus on the treatment of sprains, strains, tears, fractures, dislocation and cracks.

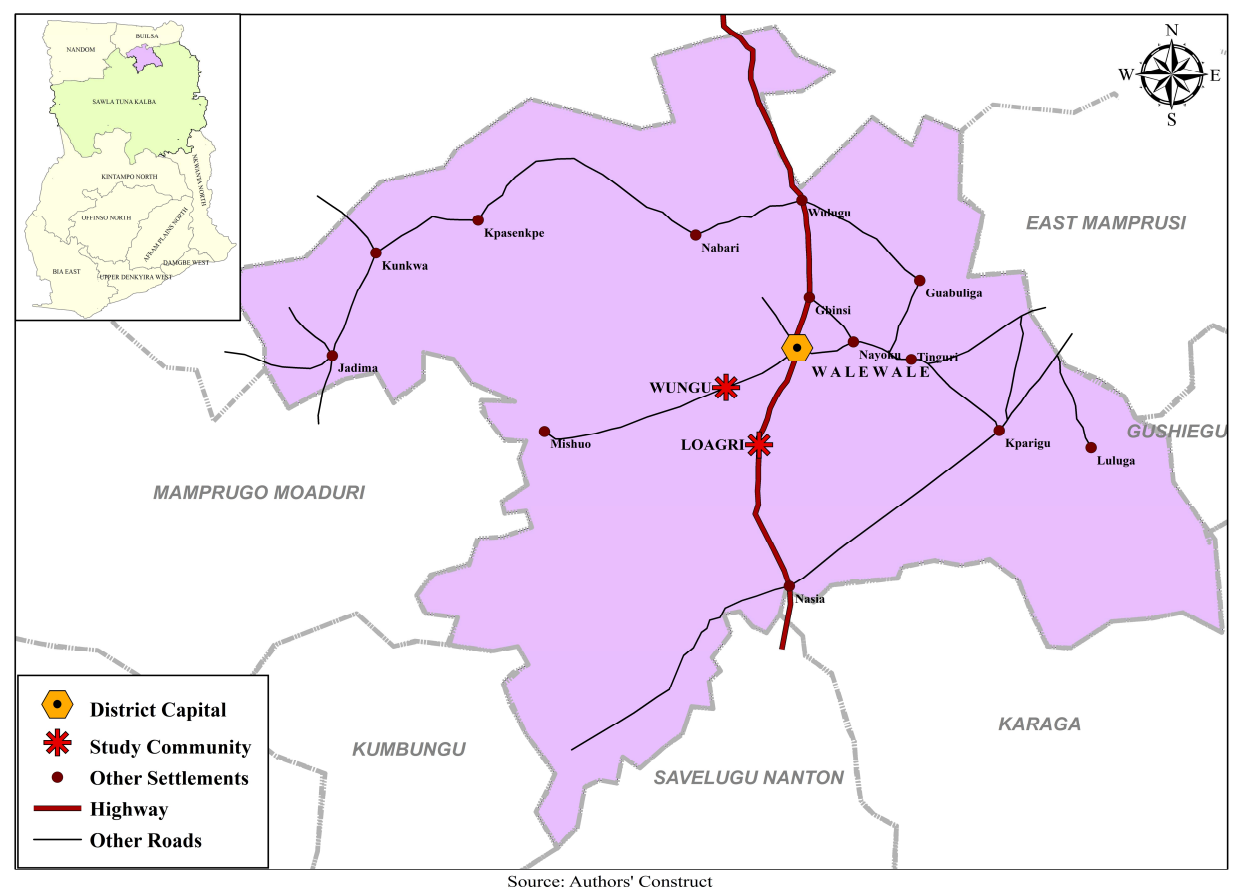

Fig. 1: Map of West Mamprusi District showing study communities 


\section{Adamtey et al.}

We adopted the case study approach by purposively selecting these two bone-setters centres who happen to be the only bone-setters in the West Mamprusi District. Data was obtained from four sources: the two bone-setters, 50 persons from each village who have had bone injuries and have been treated and discharged from each centre, 25 persons with bone injuries at each of the bone-setters centres who were receiving treatment at various stages, high profile staff of the District Hospital and the District Health Management Team (DHMT) at Walewale and 50 community residents from each of the two communities. Apart from the bonepatients who were purposively selected, the snowball technique was used to select the 50 community members from an estimated 2012 population of 7,688 (Wungu) and 1,055 (Loagri) (West Mamprusi District Assembly, 2012; 2013). Semi-structured interview guides were used in obtaining information from the various categories of respondents. Questions for both bone-patients who were receiving treatment and those who had completed treatment centered on the reasons for their choice of the kind of treatment and their level of satisfaction with the treatment. For the bone-setters, the discussions with them focused on their operations, challenges confronting them, and why they seem to be popular in healthcare delivery. With respect to the health officers at the Walewale District Hospital and the members of the DHMT interviewed, the discussion was on their views on the operations of the bone-setters in terms of their role in complementing the efforts of the District Hospital, the satisfaction of bone -patients, and issues about their relationship with the bone-setters in healthcare delivery to citizens of the district.

We adopted simple percentages to analyze the responses. Texts in terms of narratives and stories were also adopted to strengthen the results obtained. This approach seem more appropriate as it allows for in-depth analysis of the dynamics of bone setters and the sentiments of healthcare users that might not be easily obtained using other approaches such as statistical methods.

\section{FINDINGS AND DISCUSSIONS High satisfaction among persons with bone injuries}

The success rate of traditional bone-setters was judged to be very high by clients, health officers and all the community members interviewed. About $90 \%$ of those who received treatment for bone-injuries indicated that they were very satisfied with the outcome and that they opted to go to the bone-setters since they knew that they would receive the treatment needed. For those patients who were receiving treatment at various stages, all of them were very satisfied with the progress of recovery. This high level of satisfaction with the treatment as expressed by all the patients interviewed is evident in how one of the patients whose injuries were thought to be beyond treatment. He noted that:

\begin{abstract}
"Although we knew that the bone-setter could help, many people in my family and friends thought that I could not be treated and perhaps my left leg would be amputated due to the degree of injury I sustained on a hunting expedition. Apart from the scar on my leg which will give you an idea that I had an injury, you would be surprised to know that the bone at this point broke and pierced my flesh. I do not think a hospital treatment could have yielded this result. At best, my leg would have been amputated" (Personal communication with a person with bone injury-fracture, Wungu, May 2013).
\end{abstract}

The case of this person with a fractured leg was corroborated by the health officers interviewed. They indicated that given the degree of the injury and the fact that the leg could easily be infected; the orthodox treatment that might have been suggested to the patient would be amputation because it was beyond a "Plaster-of -Paris" (PoP) stage. Many of the community residents interviewed corroborated the many stories of this nature and intimated that such patients would opt for the traditional bone- 
setter instead of the hospital since they have evidence to show that the bone-setters were able to adequately and effectively treat such injuries.

Our findings support findings from similar studies on the patronage of traditional bonesetters in Maiduguri, Nigeria, by Bukar et. al., (2009:54) who found that many people who patronize the services of traditional bone-setters do so because of "the confidence they have in the therapeutic skills of the healers." The ability of bone-setters to satisfy their patients suggests that they offer quality services to bone-patients and this largely explains why planners of healthcare delivery should incorporate traditional healing services into the formal healthcare delivery system. Patients' satisfaction is an important indicator of quality of healthcare and it plays a very critical role in influencing patients' choice of health care providers (Johansson et. al., 2002; Bowers et. al., 1994). Similarly, Pascoe (1983: 185) has noted that “... patients satisfaction information can provide a dependent measure of service quality and serves as a predictor of health-related behaviour" of patients. Patient satisfaction may be measured in terms of satisfaction with medical care, satisfaction with providers, and satisfaction with outcomes of treatment (HolmesRovner et. al., 1996). The bone-patients who had completed their treatment and those under treatment were more interested in satisfaction with providers and with outcomes of the treatment.

\section{Proximity to nearest health facility}

Another major factor that influenced people's decision to either patronize the services of bone -setters or the hospital for the treatment of bone -related injuries is the proximity of the place of treatment. This largely explained the decisions of about $90 \%$ of bone-patients to patronize both Wungu and Loagri bone-setters' services. A very influential community member related the proximity issue as follows:

"Bone-injuries require immediate attention otherwise things will get out of hands and this is why bone-patients find it very convenient to go to bone-setters who are close to them. You do not need to travel very long distance and join long queues at the District Hospital in Walewale. In addition to the fact that a person with bone injury can be carried to the bone-setter's house within a very short time on a bicycle, you are also attended to immediately you arrive. Sometimes the bonesetter comes over to provide first-aid and help in the transportation of patients" (Opinion leader at Loagri, personal communication, May 2013).

All the community members from Wungu indicated that they could only access the West Mamprusi District Hospital located in Walewale, the district capital, by a six kilometer feeder road which is usually in bad condition, and unmotorable during the rainy season. Given that transport services are extremely constrained and the common means of transport are walking, motor bicycle and bicycle, it is difficult for bone-patients to be conveniently and quickly transported to the West Mamprusi District Hospital. Interviews with the bonesetters and their patients which were corroborated with vehicle operators in Walewale revealed that in rare cases, rickety taxis, mini buses and cargo vehicles ply the road but they rather exacerbate the condition of bone-patients rather than helping in transporting them.

As noted by Hardeman et al. (2004; 27) "distance to the hospital ...the quality of roads and the availability of means of transport..." all contribute to determine the quality of and access to healthcare (see also Khan, 1994; Deogaonkar, 2004; Bukar et. al., 2009). Greater distance means adding to costs in terms of transportation. Again, precious time is lost during emergencies and there is increased risk of worsening the condition of bone-patients. Interestingly, the health officers at the hospital in Walewale supported the reasons for the choice of bone-setters by bone-patients rather than reporting at the district hospital as a result of 


\section{Adamtey et al.}

the accessibility related difficulties that patients with critical bone-injuries faced.

\section{Lack of bone-setting services in the West} Mamprusi District Hospital

The West Mamprusi District Hospital is the highest health facility in the District but it has no Orthopedist to handle complex bone injuries. According to the health officers at the district hospital, bone related injuries are handled by three General Medical Practitioners: one Ghanaian medical doctor and two Cuban doctors who are not specialists to treat severe bone injuries. They added that the hospital could only attend to minor bone injuries that would only require plaster-of-Paris $(\mathrm{PoP})$ to help straighten and heal the bones.

Given the lack of specialist doctors in the West Mamprusi District to handle complex boneinjuries, it is rational for bone-patients to choose traditional bone-setters. This finding is in conformity with the WHO's finding in Uganda where the ratio of bone-setter to bonepatient is only between 1:200 and 1:400, whereas the ratio of specialist doctor in allopathic healthcare to bone-patients is $1: 20,000$ (WHO, 2002).

What this finding points to is that the unavailability of specialist services in the various District Hospitals and Health Centers across the country for the treatment of bone injuries makes the services of traditional bone-setters an important option to many citizens.

\section{The cost of treatment in hospitals}

The cost of treatment was also found to explain why over $90 \%$ of all the respondents would visit the traditional bone-setter. All the persons with bone injuries who were at various stages of treatment, those who had completed the treatment and all the community members interviewed stated that the traditional bone-setters did not charge any fee for treatment. Patients only thanked the bone-setter after he or she had recovered. The bone-setter at Wungu had this to say about why his services were free of charge:

"I was given the wisdom free of charge by our ancestors, the gods, and the spirits. As a bone-setter, I am only to help relieve the pain of society. The herbs I use are also free gift of nature. Because of all these, I am not supposed to ask for money. The patient can choose to thank me after treatment. This can be done by bringing cola nuts, fowl, or anything the patient and his family find convenient to bring. Whether such a thing is done or not is left to the patient".

This claim by the bone-setter can be a strong incentive for bone-patients to choose them. Although citizens who have registered with the National Health Insurance Scheme are supposed to be covered by the scheme, $80 \%$ of bone-patients claimed that they did not rely on the scheme because their experience is that one with bone-injuries would be given cheap medicines on the list of the NHIS drugs which are often ineffective. Health officers corroborated this and added that there are other charges for serious bone-injuries that the NHIS does not cover. These include some surgical procedures and extended stay in hospital where the patients may be required to make some payments. Most importantly, delays in the reimbursement of the District Hospital by the National Health Insurance Authority made it difficult for the hospital to deliver more effective services and to cover some types of bone injuries.

We find mounting evidence to support the suggestion that cost is a major issue in healthcare delivery in many parts of the developing world even under health insurance arrangements. For example, Deogaonkar's (2004) study on the socio-economic inequality and its effect on healthcare delivery in India found that the reduction in public health spending and the growing inequalities in health and healthcare are taking their toll on the marginalized and socially disadvantaged population. The Infant Mortality Rate among the poorest $20 \%$ of the population is 2.5 times higher than among the 
richest $20 \%$ of the population. For example, Hardeman et. al's (2004) work on access to healthcare and how user fee helps to explain access in Sotnikum (Cambodia) found that whereas households' daily earnings from field labour were between US\$0.6 and US\$0.8, they spent an average of US\$26 per month on health with the extremely poor spending US\$18 and those considered to be poor spending US\$24. In addition, the WHO (2002) noted that the relatively low cost of herbal medicines for treating ailments such as malaria largely explain the popularity and usage of traditional medicine in Kenya and Uganda where it is common practice for patients of traditional medicine to pay for treatment in kind and even according to a person's wealth.

This claim by our respondents in Wungu and Loagri brings the issue of the operation of Ghana's Mutual Health Insurance Scheme to the fore and serves as a basis to question claims about the performance of the scheme. There are claims and complaints among many Ghanaians that many health facilities across the country charge card bearers of the NHIS fees before services are rendered. Whilst some of these reports have been contested by the Ministry of Health and the National Health Insurance Authority, the issue points to a fundamental problem in Ghana's health financing system that needs scholarly debate to inform policy.

\section{The loss of patients' trust in health profes- sionals}

Many of the respondents (over 80\%) indicated that although the West Mamprusi District Hospital is highly accessible to them, they would not think about the facility as an option in the event of bone injury because they have lost confidence and trust in orthodox health professionals. They claimed that many health staff do not have the patience to listen to and attend to patients with bone injuries.

Our study revealed that patients who patronized the services of bone-setters had a lot of trust and confidence in the bone-setters. The trust seems to have been built on the testimonies of patients in respect of the nature of the good interpersonal relationship between the bonesetters and their patients. According to $75 \%$ of the patients who have received treatment for bone related injuries, the bone-setters and their aids approach the treatment process with deep emotional attachment and this gives them a lot of confidence in the treatment process. An example of the many stories told by our respondents in respect of the relationship is the one presented by a high profile professional footballer who has been treated for bone-injuries. He stated that:

"I thought my career had come to an end when I sustained the injury a little above my ankle. When I was carried to the bone-setter, his attitude and that of his aides made me realize that they were interested in treating me and not for the sake of money. All the patients at the bone-setter's house had aides to help them around the clock. They attached a lot of importance to building a good relationship with the patients by carefully and patiently explaining the treatment process to you. It is unlikely that you will find such a care in any of the hospitals that offer orthodox medicine" (A high profile professional footballer and bone-patient, personal communication, Loagri, May 2013).

All the health staff interviewed supported the views of this bone-patient. According to them, it is not always possible to give so much attention and care to the numerous patients that visit the hospitals. This may have resulted in the erosion of trust in them. Poor interpersonal relationships between healthcare givers, patients and families of patients account for the erosion of trust. According to Sheppard et al. (2004: 484), "trust is an important indicator of quality in patient-provider relationships and predicts adherence to certain protective health behaviours" and this might break down due to system failure (Etchells, 2003). Effective communication, carefully listening to patients, being caring, perceived competence and showing 


\section{Adamtey et al.}

compassion are claimed to be central to the choices patients make in terms of the health facility they chose and the health staff they are willing to take advice from (Sheppard et al., 2004; Mechanic and Meyer, 2000). When trust is dented or completely broken down, patients tend to look for help elsewhere. It seems logical that traditional bone-setters tend to attract bonepatients since in addition to their services they provide emotional support, care and compassion that seem to be lacking in many hospitals across Ghana. But this may be so as many health staff in public hospitals are poorly motivated to work and are highly frustrated (Connor, 2009; Agyepong et. al., 2004). This is evidenced by many industrial actions embarked upon by health professionals in recent times and the rise in migration among health personal out of Ghana.

\section{Socio-cultural norms and belief system}

The WHO (2002: 20) has noted that "traditional medicine is also highly popular in many developing countries because it is firmly embedded within wider belief systems." A study by Uba (1992) on cultural barriers to health care for southeast Asian refugees in the United States found that in spite of the numerous health problems of the refugees, they underuse available health facilities due to cultural reasons: the belief that human suffering and pain due to ill-health is part of human life and that death is normal as how long one can live is predetermined. In fact, their lack of familiarity with the diagnostic techniques and treatment procedures make them apprehensive. Interestingly, Jenkins et. al. (1996) did not find sociocultural attributes (traditional beliefs and practices) as explanatory factors of minimal utilization of health services by Vietnamese immigrants in the San Francisco Bay area in the United States of America.

In our study, it was revealed that the treatment of bone-injuries is heavily rooted in superstition and beliefs. The bone-setters employ the use of taboos and incantations, a very common feature of this type of healers (Bukar et. al., 2009).
According to all the patients, one is asked to observe certain practices that are laden with rituals during the course of the treatment such as fasting or abstinence from alcoholic beverages. The nature of the belief and superstition around the treatment of bone-injuries was given by the bone-setter at Wungu as:

"Many of the bone-injuries are as a result of offences committed against the gods and other spirits so we need to pacify the gods through certain rituals to be able to treat you. In many cases we need the permission of some spirits to be able to offer treatment especially when it comes to the herbs we use. We need to collect some of the herbs, roots and barks at midnight so that no human being will see them. If this is not done, it is possible that you will not find the herb in the afternoon or it will not be effective if you are seen picking them. With some injuries we use animals and insects. The herbs and the animals are spirits themselves" (Bone-setter, personal communication, Wungu, May 2013).

All the staff at the West Mamprusi District Hospital we interviewed would not accept the superstitious claims made by the bone-setters in terms of the causes of bone-injuries and the treatment procedure. In their view, boneinjuries are due to accidents and not gods or spirits. They also disagree that the herbs or leaves are spirits. They held the view that the treatment process is shrouded in superstition in order to keep the treatment procedures and skills secret to themselves and their families so that they can monopolize the art.

In spite of the diverse views about how sociocultural norms help explain healthcare behaviour of many Africans, what this finding points to is that culture is important and may help us, to some extent, understand and plan health service utilization in the wider society in Ghana. This is supported by Rothschild's (1998: 2930) statement that "physicians who actively seek to understand their patients' culture will find their 
Traditional bone-setters and rural healthcare delivery...

simple efforts amply rewarded [in the form of] increased patients' satisfaction and greater clinical effectiveness."

Although all the factors were found to influence patient's choices, there were good indications that all of them could not be said to have the same weight and importance to patience. Proximity to the bone-setters and the cost of treatment were ranked highest among the factors. What this suggests is that the conventional processes of planning the equitable distribution of health facilities to cover deprived regions in the country and the effectiveness of the health insurance scheme need to be revisited by central government.

Although traditional bone-setting services were found to play a very important role in general healthcare delivery in deprived regions within the West Mamprusi District and surrounding communities, a number of issues emerged that seem to hamper the performance of these services. Rural health services planning needs to consider these challenges carefully.

\section{CHALLENGES OF TRADITIONAL BONE -SETTERS}

Weak collaboration with West Mamprusi District Hospital

We found weak collaboration between bonesetters in both Wungu and Loagri on the one hand and the West Mamprusi District Hospital on the other hand. A number of reasons were given by the bone-setters and hospital staff to explain this. First, a high profile hospital staff indicated that the bone-setter clinics operate without any registration or license. Second, they use traditional splints which the hospital staff claim can cause severe infection of wounds, gangrenes, short limbs and septicemia. Although the hospital staff mentioned that this was not the case in Loagri as the bone-setter uses bandages and gauze which result in little and sometimes no infections, the lack of hygienic environment exposes patients to infection during the application of the bandages or gauze.
On the part of the bone-setters, many hospital staff do not have confidence in their services and so there is no incentive to think about collaborating with them. This is very interesting and contrasts with collaboration between traditional medical practitioners and their counterparts in orthodox medicine in countries such as the People's Republic of China. According to the WHO (2002: 8-9), the Democratic People's Republic of Korea, the Republic of Korea and Vietnam are examples of countries that operate an integrative system in which traditional medicine is officially recognized and incorporated into all areas of healthcare provision. Traditional medicine is included in national health and drug policy and both healthcare providers and their medicines have been registered and regulated by the health ministry. It is possible for patients to access traditional medicine in both public and private health facilities and they are covered by health insurance.

In spite of the issues raised by bone-setters and the health staff, we found some level of collaboration between them though weak. The hospital sends nurses to educate the bonesetters especially the one in Loagri on how to use some simple modern healthcare materials like bandage and gauze.

Interestingly, our interviews with opinion leaders, the bone-setters and community members revealed the bone-setting clinics rather collaborate with a number of Non-governmental organizations (NGOs), agencies and institutions. These institutions are Neighbour in Need Foundation (NENFOUND), the West Mamprusi District Assembly (WMDA), Trent University, Innovations for Poverty Reduction (IPA), and the Loagri community which contribute in diverse ways in the building and maintenance of the accommodation facilities of the bone-setter clinics. Northern Region Poverty Reduction Programme (NORPREP), International Fund for Agricultural Development (IFAD) and the West Mamprusi District Assembly help in the provision of food, crutches, mattresses, gauze and bandages to the clinics. 


\section{Adamtey et al.}

\begin{abstract}
Absence of modern equipment and materials Both bone-setting clinics did not have imaging equipment such as X-Ray facility, which could be used to detect and diagnose the type and extent of damage to patients' bones. They only use their fingers to determine the damage and extent of injury. Clearly, it cannot be said that bone-setters have scientific knowledge of the human anatomy as we found that none of them had formal education or training in any field related to the human anatomy. Even if patients are to take X-Ray photographs from other health facilities, they stand the chance of not accurately interpreting the photographs. In addition, bone-setters and their aids did not use gloves when attending to patients with open fractures and sores around the fractures. They use their bare hands putting the patient at risk of infection and also standing the chance of infecting themselves. Although this method might not be very reliable and unscientific, it has been the practice. This partly explains why orthodox medical practitioners would not approve the treatment of complex and complicated fractures by bone-setters.
\end{abstract}

\section{No record keeping}

The Loagri bone-setter has no record keeping system to take care of the record keeping needs of the clinic. The records of patients who attend the clinic are not properly organized and kept so that such records can be used in monitoring the progress of patients and also relied upon in the future when the patients attend the clinic. In addition, the bone-setters cannot tell the number of people they take care of unless they take a head count and that can only be possible for the in-patients.

Inadequate accommodation to house inpatients

The accommodation for the clinics is not adequate for the patient as claimed by the bonesetters and all the patients. According to the bone-setters, although they receive several patients with serious injuries that need to be admitted and treated, they are unable to do so because of inadequate accommodation for them. The bone-setter in Wungu has 5 detached huts with each accommodating 4 patients over the treatment period between 3 to 9 months. This was an issue of concern to both the bonesetter and their patients as indicated by the bone -setter that:

"Ideally I would like to put two patients in each hut so that each patient will have one family member to stay with him/her to attend to their personal needs such as washing down and supported to do simple exercises that are essential in the treatment process. Unfortunately this is not so leading to the early discharge of many of the patients to make way for others" (Bone-setter, Wungu, personal communication, May 2013).

This story was not different from our findings from the interviews with patients and the bonesetter at Loagri. In both cases, water and toilet facilities were found to be inadequate.

\section{No reliable source of income for the clinic}

What may have worsened the plight of the bone -setters to provide accommodation is that their services are for gratis. Both bone-setters in Wungu and Loagri claimed that they did not charge patients for the services rendered to them. They only receive gifts of appreciation from some of the patients after they have been treated. Because many cannot afford, the gratis nature of the services attracts several patients. This makes the revenue of the clinics inadequate. They indicated that they depend on donors to provide some of the needs of the clinic like food, crutches, bandages and gauze.

\section{POLICY RECOMMENDATIONS}

Effective collaboration with district hospitals The weak collaboration between the bone-setter clinics and the West Mamprusi District Hospital and the various Community-based health planning services (CHPS) compounds in the district needs to be strengthened through effective planning. The Ministry of Health should liaise with the Ghana Federation of Traditional Medicine (GAFTRAM) of which the bone- 
setters are members so that an inventory of all bone-setters in the Northern part of Ghana could be taken. They should then be given training in basic hygiene and prevention of infections. They should be educated and encouraged to refer cases which are beyond their expertise to a health facility. One way to do this effectively will be for health staff to pay monthly visits to the bone-setters to ensure that the environment in which patients are treated, the materials used and all other practices are within the basics for proper healthcare delivery.

This collaboration seems critical as $84 \%$ of the patients of traditional bone-setters were found to be taking other orthodox medicine especially pain-killers alongside the treatment of the traditional bone-setter because they had the view that forging concerted efforts between traditional bone-setting and orthodox medicine might enhance treatment of bone injuries.

\section{Support from the West Mamprusi District Assembly}

The West Mamprusi District Assembly needs to take into consideration the services of these bone-setters when preparing development plans for healthcare delivery in the District by facilitating their training, monitoring, registration and licensing with the Ministry of Health and making contributions in the provision of materials and equipment that they need in order to deliver improved services.

\section{Integration of traditional medicine into pub- lic health system}

Rural health care planning must pay more attention to the inclusion of topics on traditional medicine such as bone-setting in the training of medical students as is done elsewhere such as China where elements of Chinese medicine (which includes manipulation, massage and relaxation) are included in the curricular of conventional health workers training. The department of herbal medicine at the Kwame Nkrumah University of Science and Technology in Kumasi should forge a partnership with the bone-setters and the Mampong Centre for
Research into Plant Medicine. A mutual collaboration between them might help to promote the work of bone-setters and enhance research into herbal medicine.

\section{CONCLUSION}

The study has shown that traditional bonesetting services as in Wungu and Loagri are very important components of national healthcare delivery. These services will remain crucial in the lives of both rural and urban dwellers given the fact that many Ghanaians use both traditional and orthodox health services. These realities should provide lessons that rural health planning will build upon.

\section{REFERENCES}

Agarwal, A. and Agarwal, R. (2010).The Practice and Tradition of Bone setting. Education for Health, 23(1): 225-233.

Agyepong, I. A., Anafi, P., Asiamah, E., Ansah, E. K., Ashon, D. A. and Narh-Dometey, C. (2004). Health worker (internal customer) satisfaction and motivation in the public sector in Ghana. International Journal of Health Planning and Management, 19 (4):319-36.

Bowers, M. R., Swan, J. E. and Koehler, W. E. (1994). What attributes determine quality and Satisfaction with health care delivery? Health care Management Review, 19(4): 49-55.

Bukar, N. U., Umar, A., Umar, J. N., Lawal, A., and Ngohi, M. B. (2009). Causes of Bone Injuries and Patronage of Traditional Bone Setters in Maiduguri, Nigeria. Sierra Leone Journal of Biomedical Research, 1(1): 50-54.

Connor, C. (2009). Strengthening Health Systems to Reach the MDGs (Breaking with Business as usual: MDG Strategic Conference-MIDEGO) Available at: http:// w w w.health s y t e m s 2020 . org/ files/2556_file_Strengthening_Health_Syste $\mathrm{ms}_{-} \mathrm{to} \mathrm{R}_{-} \mathrm{Reach}$ the $\mathrm{MDG}_{-} \mathrm{v}_{-} 3_{-} \cdot \mathrm{ppt}$ (accesses on 05/08/2013) 
Darimani, B (2007). "Photographic Documentation and Description of the Arts and Activities of the Traditional Bone-Setters' Clinic of Gwollu", Unpublished Thesis for the Award of Master of Philosophy, African Arts and Culture, Faculty of Fine Arts, College of Arts and Social Sciences, KNUST, Kumasi, Ghana.

Deogaonkar, M. (2004). Socio-economic inequality and its effect on healthcare delivery in India: Inequality and healthcare. Available athttp://www.sociology.org/ content/vol8.1/deogaonkar.html(accessed on 7th. May 2014).

Etchells, R. (2003). The Patient's Perspective. In Harrison, J., Innes, R. and van Zwanenberg, T. (eds.) Rebuilding Trust in Healthcare. Radcliffe Medical Press Ltd., London pp 5-14

Hardeman, W., Van Damme, W., Van Pelt, Por, I., Kimvan, H. K. and Bruno, M. (2004) Access to healthcare for all? User fees plus a Health Equity Fund in Sotnikum, Cambodia. Health Policy and Planning, 19(1):22-32.

Holmes-Rorner, M., Kroll, J., Schmitt, N., Rovner, D. R., Breer, M. L., Rothert, M. L., Padonu, G. and Talarczyk, G. (1996). Patients satisfaction with health care decisions: the Satisfaction with decision scale. Medical Decision Making, 16(1): 58-64.

Jenkins, C. H. N., Thao, L., McPhee, S. J., Stewart, S. and Ngoc, T. H. (1996). Healthcare access and preventive care among Vietnamese immigrants: Do traditional beliefs and practices pose barriers? Social Science and Medicine, 43(7): 1049-1056.

Johansson, P., Oleni, M. and Fridlund, B. (2002). Patients satisfaction with nursing care in the Context of health care: a literature study. Scandinavian Journal of Caring Sciences, 16(4): 337-344.
Kavi, E., Abanga, R. A., Kudolo, P. A. and Morna, E. (2000). Traditional Medicine Project: A Survey on the Practice of Traditional Medicine in the Operational area of Eight ACDEP-Member Primary Health care Programmes in the Upper East and Northern Regions of Ghana. Tamale, ACDEP and CORDAID. Available on 04/02/15 from http://acdep.org/wordpress/wp-content/ uploads/2010/12/TMP-Report2.pdf

Khan, A. A. (1994). Access to Health Care: A Conceptual Framework and its Relevance to Health Care Planning. Evaluation and the Health Professions, 17(1): 60-76.

Marcel, J. H. A., Hanneke, J., Wegdam, H. H. J. and Geest, S. V. D. (2007). Fracture treatment by bonesetters in central Ghana: patients explain their choices and experiences, Tropical Medicine and International Health, 12 (4): $564-574$

Mechanic, D. and Meyer, S. (2000). Concepts of trust among patients with serious illness. Social Science and Medicine, 51(5): 657668.

Ministry of Health (1995). Traditional and Modern Health care: Partnership for the Future: A Report on National Consensus, Ministry of Health, Accra.

Olayiwola, A. (1987). The best of both worlds: Bringing Traditional Medicine up to date. Social Science and Medicine, 24(2): 177-181

Osemwenkha, S. (2000). Disease Aetiology in Traditional African Society. Revistatrimestrade distudie documentazione dell' Istitutoitaliano per I'Africa e I'Oriente, 55(4): 583-590

Pascoe, G. C. (1983). Patients satisfaction in Primary Health care: A literature review. Evaluation and Program Planning, 6(3-4): $185-210$ 
Traditional bone-setters and rural healthcare delivery...

Republic of Ghana (2007). National Health Policy: Creating Wealth through Health, Accra, Ministry of Health

Republic of Ghana (2005a). Growth and Poverty Reduction Strategy (GPRS II) (20062009), Vol. 1, Policy Framework, Accra, National Development Planning Commission

Republic of Ghana (2005b). Policy Guidelines on Traditional Medicine Development, Accra, Ministry of Health

Republic of Ghana (1997). Ghana-Vision 2020: The First Medium-Term Development Plan (1997-2000), Accra, National Development Planning Commission

Rothschild, S. K. (1998). Cross-cultural issues in Primary care medicine. Disease-a-Month, 44(7): 293-319.

Shepppard, B. V., Zambrana, R. E. and O'Malley, A. S. (2004). Providing healthcare to low-income women: a matter of trust. Family
Practices, 21(5): 484-491

Uba, L. (1992). Cultural barriers to health care for Southeast Asians refugees. Public Health Reports, 107(5): 544-548.

United Nations Development Programme (2005). Investing in Development: A Practical Plan to Achieve the Millennium Development Goals. New York, UNDP

West Mamprusi District Assembly (2012). Poverty Mapping Study (Unpublished), Walewale, West Mamprusi District Assembly

West Mamprusi District Assembly (2013). Agricultural Production and Poverty (Unpublished), Walewale, West Mamprusi District Assembly

World Health Organization (2002), World Health Report. Traditional Medicines Strategy 2002-2005 World Health Report WHO, Geneva 